\title{
Laparoscopic Sacrocolpopexy - A Retrospective Study
}

\author{
Kavitha D Yogini ${ }^{1 *}$, Rashmi Barnwal ${ }^{2}$, Devi Balasubramaniam ${ }^{3}$ and Chinnusamy Palanivelu ${ }^{4}$ \\ ${ }^{1}$ HOD of Endogynecology Department, Gem hospital, India \\ ${ }^{2}$ Endogynecology Department, Gem Hospital, India \\ ${ }^{3}$ Consultant, Gem Hospital, India
}

${ }^{4}$ Director and Head, Gem Hospital, India

Received: October 19, 2017; Published: October 25, 2017

*Corresponding author: Kavitha D Yogini, HOD, Endogynecology, Gem hospital, Coimbatore, Tamilnadu, India; Tel: 8883112537;

Email: yoginianandvij@rediffmail.com

\begin{abstract}
Post-hysterectomy vaginal vault prolapsed is a common complication following different types of hysterectomy which can lead to significant urinary, anorectal and sexual impairment in the patients. Pre existing pelvic floor defect is the most important predicting factor in the future development of vaginal vault prolapse. Preventive measures can be taken intraoperatively to reduce the incidence of later development of vault prolapse but the possibility cannot be eliminated completely. Both vaginal and abdominal approaches have been advocated for the correction of vault prolapse. With the abdominal approach, the results are better and recurrence is less. However, recovery time is more and the procedure requires a lot of bowel manipulation. Laparoscopic sacrocolpopexy can circumvent the disadvantages of open abdominal approach while retaining its advantages of great patient satisfaction and less recurrence. Being a minimally invasive procedure, lap sacrocolpopexy allows for less blood loss and speedy recovery. Here, we present a retrospective analysis of 25 cases of laparoscopic sacrocolpopexy over 2 years.
\end{abstract}

Keywords : Laparoscopy; Vault Prolapsed; Sacrocolpopexy; Polypropelene Mesh

\section{Introduction}

The incidence of vault prolapsed in post hysterectomies patients is estimated to be $0.2-44 \%[1,2]$ and is especially common in parous patients with vaginal deliveries and in patients who have previously undergone hysterectomy for uterine prolapsed $[3,4]$. The risk increases even more in patients who have undergone previous hysterectomy in view of prolapse uterus [5]. Numerous surgical procedures are described for the management of vault prolapse. Vaginal repair is associated with less postoperative pain and early recuperation as compared to abdominal approach. However, abdominal sacrocolpopexy has better anatomical outcome [6]. Laparoscopic sacrocolpopexy seems to be a promising approach which can provide the result of abdominal sacrocolpopexy with decreased morbidity. The success rate of laparoscopic sacrocolpopexy has been reported by a number of authors to be 90-98\% [7]. However, the limitation of lap sacrocolpopexy is that it requires extensive dissection and advanced suturing skills.

\section{Materials and Methods}

25 women with vault prolapse who underwent laparoscopic sacrocolpopexy over a period of 2 years at our institute (September 2013 to October 2015) were included in this study. Parameters recorded include age, parity, BMI, previous pelvic surgery ; its indication and route, concomitant surgical procedure, duration of surgery, estimated blood loss, duration of hospital stay and postoperative minor and major complications. All patients were assessed clinically and prolapsed was graded on the Baden- Walker scale at all sites preoperatively as well as postoperatively during follow up at 1 week, 3months, 6 months and 1 year. Preoperatively and at each postop follow up, patient was also asked to fill a standard Pelvic floor distress inventory questionnaire for subjective assessment of the prolapse.

\section{Technique}

All patients were evaluated thoroughly preoperatively. Good bowel preparation was ensured by keeping the patient on 2 days of liquid diet and giving exelyte enema on the previous night of surgery. Prophylactic dose of antibiotics was given just prior to surgery. After creating pneumoperitoneum, a $10 \mathrm{~mm}$ camera port was placed supraumbilically and three $5 \mathrm{~mm}$ ports were placed, one each in iliac fosse and third one between symphysis and umbilicus. On entry, approach to the vault was assessed. In case of vault adhesions, adhesiolysis was done and vault exposed. The vaginal assistant placed CCL extractor to lift up the vault to aid in dissection. Incision was given on the vault so as to open up the uterovesical fold of peritoneum and bladder was carefully dissected 
down so as to expose the vagina sufficient enough for anterior fixation of the mesh. Similarly, rectovaginal space was entered posterior and rectum was dissected down. The peritoneum over sacral promontory was incised after identification of L5-S1, left common iliac vein and right ureter.

Dissection was carried out retroperitoneal till anterior sacral ligament was exposed. Care was taken not to injure median sacral vessels. Right ureter was traced from the pelvic brim along the lateral pelvic wall to its entry into broad ligament. A tunnel was created retroperitoneally along the rectosigmoid staying medial to the ureter, opening the rectovaginal space. A Y shaped polypropylene mesh was anchored to the vaginal vault using prolene 2-0. Approximately 3-5 sutures each were placed on the vault to fix the anterior and posterior limb of the Y shaped mesh. The vertical limb of the mesh was taken retroperitoneal and fixed to the anterior sacral ligament at the level of sacral promontory (after pushing and positioning the vault at the level of ischial spine). The exposed peritoneum over the vault and sacral promontory was closed with continuous sutures of vicryl 2-0.

\section{Results}

Table 1: Patient Charcteristics: Median.

\begin{tabular}{|c|c|}
\hline Age of patients & $\mathbf{6 0}$ years (45-73 years) \\
\hline BMI (Body Mass Index) & $35(26-40)$ \\
\hline Parity & $2(1-5)$ \\
\hline
\end{tabular}

Table 2: Level of Defect.

\begin{tabular}{|c|c|}
\hline Level i defect (vault \pm enterocele) & 19 \\
\hline Level i+ii defect (vault+cystocele) & 05 \\
\hline $\begin{array}{c}\text { Level } \text { i+ii+iii } \\
\text { defect(procidentia+cystocele+rectocele) }\end{array}$ & 01 \\
\hline
\end{tabular}

Table 3: Type of Previous Hysterectomy.

\begin{tabular}{|c|c|}
\hline Vaginal Hysterectomy & 14 \\
\hline Lavh & 03 \\
\hline Total Abdominal hysterectomy & 06 \\
\hline Total Laparoscopic hysterectomy & 01 \\
\hline
\end{tabular}

Table 4: Concomitant Procedures.

\begin{tabular}{|c|c|}
\hline Lap BSO & 15 \\
\hline Lap Paravaginal repair & 4 \\
\hline Anterior Colporrhaphy & 2 \\
\hline Posterior Colpoperineorrhaphy & 1 \\
\hline Lap Ipom & 1 \\
\hline
\end{tabular}

25 women with a median age of 60 years were studied (range 45-73 years) (Table 1). All patients were multipara and had normal vaginal deliveries, one patient had forceps delivery. The major presenting complaint was mass descending per vaginum. All patients had previously undergone hysterectomy except one who presented to us with huge procidentia and hysterectomy was done at the time of sacrocolpopexy (Table 2). 17 patients had previously undergone vaginal or lap assisted vaginal hysterectomy for uterovaginal prolapse while 6 patients had history of abdominal hysterectomy, one patient had history of total laparoscopic hysterectomy (Table 3). Preoperatively, all women had a vault descent greater than or equal to stage 2, as graded on Baden-Walker system. 15 patients had concomitant procedures performed at the time of laparoscopic sacrocolpopexy. Bilateral salpingo oophorectomy was done concomitantly in 15 patients while 7 patients required concomitant anterior or posterior compartment repair (Table 4). Initially we used to do cystocele repair vaginally, but later with better and evolved technique we are doing laparoscopic paravaginal repair for anterior compartment repair.

The mean duration of sacrocolpopexy procedure was 45 mins excluding the time required to do any concomitant procedure. Intraoperatively, there were no major complication, in one case we were faced with bleeding from median sacral vessels which was taken care of by placing a suture ligature (Table 5). Postoperatively, all patients had uneventful recovery. All patients were followed up at 1 week, 3 month, 6 month and 12 month. One patient presented with fever and chills 10 days after surgery and USG showed a $3.6 \times 2.9 \times 2.5 \mathrm{~cm}$ collection around the mesh in the pelvis posterior to bladder. Patient was treated with 5 days of injectable antibiotics and subsequent scan after 7 days showed no collection in pelvis (Table 6).

Table 5: Intraoperative Complication.

\begin{tabular}{|c|c|}
\hline Middle Sacral Artery Bleeding & 1 \\
\hline Bladder Injury & 0 \\
\hline Bowel Injury & 0 \\
\hline
\end{tabular}

Table 6: Postoperative Complication.

\begin{tabular}{|c|c|}
\hline Immediate (within 48 hours) & Nil \\
\hline Delayed (after 48 hours) & 1 (collection around mesh) \\
\hline
\end{tabular}

At 3 month follow up, one patient complained of vaginal discharge which responded to topical estrogen and oral antibiotics. One patient had complaint of urge incontinence preoperatively which persisted postoperatively, while another one patient developed de novo urge incontinence at 3 month follow up which settled down afterwards with bladder training exercises (Table 7). There was no other major or minor complication during follow up of rest of the patients.

Table 7: Follow Up Visits.

\begin{tabular}{|c|l|}
\hline Stress Urinary Incontinence & 0 \\
\hline Persistent Urge Incontinence & 1 \\
\hline De novo Urge Incontinence & 1 \\
\hline Postoperative Prolapse & 0 \\
\hline Bowel Symptom & 0 \\
\hline Vaginal Discharge & 1 \\
\hline
\end{tabular}

\section{Discussion}

Vaginal vault prolapse can significantly affect quality of life by causing symptoms of pressure and discomfort, and by its effect on 
urinary, bowel and sexual function. The risk of future vault prolapse is six-fold higher if the initial indication for hysterectomy was for prolapse compared with other indications, such as menorrhagia or pelvic pain $[1,8]$. Current treatment options include pelvic floor muscle training, use of pessaries and surgery. Surgical treatment should be offered to women with symptomatic vault prolapse after appropriate counselling. A variety of procedures exist for surgical treatment of vault prolapse and the type of operation performed should be tailored to the individual case, such as concomitant prolapse in other compartment(s), previous abdominal surgery, previous prolapse surgery, the total vaginal length, and associated comorbidities. Sacrocolpopexy using mesh for vaginal vault prolapse repair is performed with the patient under general anesthesia, using an open or laparoscopic abdominal approach. Mesh is attached to the apex of the vagina and may also be attached to the anterior and/or posterior vaginal wall.

Several different types of synthetic and biological mesh are available, which vary in structure and in their physical properties such as absorbability. Transabdominal sacrocolpopexy is a definitive treatment option for vaginal vault prolapse with durable success rates [2,9]. However, it is associated with increased morbidity compared with vaginal repair [10-12]. Laparoscopic sacrocolpopexy can be equally effective as abdominal sacrocolpopexy in selected women with vault prolapse and can be combined to include mesh extension or be combined with other vaginal procedures to correct other compartment prolapse. A number of observational studies have shown good anatomical cure rates (more than 90\%) in women undergoing LSC at 1-2 years' follow-up [13-15]. Beer et al. [16] showed that objective and subjective cure rates are not necessarily the same; subjective cure rate being determined by functional outcome rather than anatomical result [16]. We also tried to evaluate both subjective rates (patient's satisfaction) and objective rates in our study. We had excellent vault support in all 25 cases on follow up. We did not come across any major intraoperative complication, in one case where median sacral vessel started bleeding, a suture ligature was placed and bleed was controlled. At 3 month follow up none of our patients had any significant complaint except for one who complained of vaginal discharge.

All patients were fully satisfied with the procedure and there was no complaint of any symptom of prolapse. In our study, there was no case of suture erosion though we used non absorbable prolene 2-0 sutures. Also, there was no mesh erosion during follow up; however, one patient had a small collection around the mesh at 10th postoperative day which got settled with 5 days parenteral antibiotics. Noushin et al in their study concluded that there is no need of concomitant prophylactic surgery for SUI at the time of LSCP in women who do not have objectively demonstrable SUI before surgery [17]. Hence, in our study there was no prophylactic SUI surgery and follow up cases did not show any postoperative overt SUI.

Need of peritonising the mesh was obviated in our technique as we, instead of opening the peritoneum along the entire length of recto sigmoid, made a tunnel through which the vertical limb of Y shaped mesh was passed. This particular step also saved time as peritoneal closure was not needed at the end of the procedure. In our study, we evaluated both anatomical cure and functional outcome, which was $100 \%$. However, the only limitation of our study is the less number of patients and a relatively short follow up.

\section{Conclusion}

The laparoscopic sacrocolpopexy is a minimally invasive technique for vaginal vault prolapse repair, combining the advantages of open sacrocolpopexy with the decreased morbidity of laparoscopy. We found low complication rates, early recuperation and high rate of objective and functional outcome with a minimum of 1 year follow-up.

\section{References}

1. Marchionni M, Bracco GL, Checcucci V (1999) True incidence of vaginal vault prolapsed:Thirteen years of experience. J Reprod Med 44(8): 679-684.

2. Flynn BJ, Webster GD (2002) Surgical management of apical vaginal defect. Curr Opin Urol 12(4): 353-358.

3. Mant J, Painter R, Vesey M (1997) Epidemiology of genital prolapse: observations from the Oxford Family Planning Association Study. Br J Obstet Gynaecol 104(5): 579-585.

4. Altman D, Falconer C, Cnattingius S, Granath F (2008) Pelvic organ prolapse surgery following hysterectomy on benign indications. Am J Obstet Gynecol 198(5): 572e1-572e6.

5. Dällenbach P, Kaelin Gambirasio I, Dubuisson JB, Boulvain M (2007) Risk factors for pelvic organ prolapse repair after hysterectomy. Obstet Gynecol 110(3): 625-632.

6. Benson JT, Lucente V, McClellan E (1996) Vaginal versus abdominal reconstructive surgery for the treatment of pelvic support defects: a prospective randomized study with long term outcome evaluation. Am J Obstet Gynecol 175(6): 1418-1421.

7. Agarwala N, Hasiak N, Shade M (2007) Laparoscopic sacral colpopexy with gynemesh as graft material: experience and results. J minim Invasive Gynecol 14(5): 577-583.

8. Symmonds RE, Williams TJ, Lee RA, Webb MJ (1981) Posthysterectomy enterocele and vaginal vault prolapse. Am J Obstet Gynecol 140: 852859.

9. Illiano E, Giannitsas K, Zucchi A, Di Biase M, Del Zingaro M, et al. (2016) Sacrocolpopexy for posthysterectomy vaginal vault prolapse: long-term follow-up. Int Urogynecol J 27(10): 1563-1569.

10. Nygaad IE, McCreery R, Brubaker L (2004) Abdominal sacrocolpopexy: a comprehensive review. Obstet Gynaecol 104(4): 805-823.

11. Maher CF, Qataweh AM, Dwyer PL (2004) Abdominal sacrocolpopexy or vaginal sacrospinous colpopexy for vaginal vault prolapse : a prospective randomized study. Am J Obstet Gynecol 190(1): 20-26.

12. Elliott DS, Krambeck AE, Chow GK (2006) Long term results of robotic assisted laparoscopic sacrocolpopexy for the treatment of high grade vaginal vault prolapse. J Urol 176(2): 655-692.

13. Claerhout F, De Ridder D, Roovers JP, Rommens H, Spelzini F, et al. (2009) Medium-term anatomic and functional results of laparoscopic sacrocolpopexy beyond the learning curve. Eur Urol 55: 1459-1467.

14. Klauschie JL, Suozzi BA, O’Brien MM, McBride AW (2009) A comparison of laparoscopic and abdominal sacral colpopexy: objective outcome and perioperative differences. Int Urogynecol J Pelvic Floor Dysfunct 20(3): 273-279.

15. Price N, Slack A, Jackson SR (2011) Laparoscopic sacrocolpopexy: an observational study of functional and anatomical outcomes. Int Urogynecol J 22(1): 77-82. 
16. Beer M, Kuhn A (2005) Surgical techniques for vault prolapse: a review of the literature. Eur J Obstet Gynecol Reprod Biol 119(2): 144-155.
17. Noushin Khoshbakht, May Alarab, Danny Lovatsis (2012) Stress Urinary Incontinence Six Months Post Laparoscopic Sacrocolpopexy. Journal of Obstetrics and Gynaecology Canada 34(7): 653-656.

BIOMEDICAL
RESEARCIIES $\quad \begin{aligned} & \text { Assets of Publishing with us } \\ & \text { - Global archiving of articles }\end{aligned}$

